DOI $10.35381 / \mathrm{cm} . v 5 i 9.213$

\title{
Estrategias de verificación del montaje de un sistema electrónico usando Técnicas de Boundary-Scan (JTAG)
}

\section{Verification strategies for the assembly of an electronic system using Boundary- Scan Techniques (JTAG)}

\author{
Alba de los Cielos Miranda Villacis \\ albamiranda@uta.edu.ec \\ Universidad Técnica de Ambato, Ambato \\ Ecuador \\ https://orcid.org/0000-0003-4268-4116 \\ Marlon Ernesto Moscoso Martínez \\ emoscoso.ing@gmail.com \\ Universidad Técnica de Ambato, Ambato \\ Ecuador \\ https://orcid.org/0000-0001-5182-3099 \\ Miguel Andrés Sánchez Almeida \\ andyplott@hotmail.com \\ Universidad Técnica de Ambato, Ambato \\ Ecuador \\ https://orcid.org/0000-0003-4858-284X
}

\begin{abstract}
RESUMEN
El actual artículo de revisión analizó las estrategias de verificación del montaje de un sistema electrónico usando Técnicas de Boundary-Scan (JTAG). La estrategia para el aprendizaje JTAG, es necesario basarla en la generación de proyectos de investigación, para esto se requiere que el estudiante sea formado en una visión integral desde una concepción metodológica. El mundo de los sistemas JTAG, abre la posibilidad de transcender la visión educativa tradicional para trabajar por proyectos, esto implica que se pueden conformar equipos de trabajo en la universidad, donde puedan participar diversos docentes y estudiantes, bridando una noción multidisciplinaria al proyecto.
\end{abstract}

Descriptores: Medios electrónicos; Codificación; Red informática; Aplicación informática. 


\begin{abstract}
The current review article analyzed the verification strategies for the assembly of an electronic system using Boundary-Scan Techniques (JTAG). The JTAG learning strategy, it is necessary to base it on the generation of research projects, for this it is required that the student be trained in an integral vision from a methodological conception. The world of JTAG systems, opens the possibility of transcending the traditional educational vision to work for projects, this implies that work teams can be formed in the university, where various teachers and students can participate, providing a multidisciplinary notion to the project.
\end{abstract}

Descriptors: Electronic media; Encoding; Computer networks; Computer applications.

\title{
INTRODUCCIÓN
}

El Boundary-Scan (JTAG), permite el escaneo de posibles errores en dispositivos digitales, de ese modo, se puede ejecutar la corrección de los mismos, mediante la lectura encriptada de los códigos del dispositivo, en este sentido la empresa Ikor (2015), señala que:

El sistema de test "Boundary-Scan", también conocido como "JTAG" es una técnica de ensayo de "escaneo de límites" en sistemas electrónicos para circuitos integrados o chips (microprocesadores, microcontroladores, memorias, chipsets, etc...) Para ello, estos deben ser compatibles con este sistema con el objeto de poder verificar sus conexiones eléctricas que, por su formato encapsulado, no están accesibles físicamente a los sistemas de test tradicionales. De esta forma se puede reducir el tiempo de testeo, de depuración y además aumentar la fiabilidad (p. 1).

Esto origina efectividad en el trabajo realizado por parte del técnico, dado que la versatilidad del sistema le posibilita la reparación de errores que difícilmente serían reparados sin el sistema JTAG. Es así que se realiza una revisión de los aportes de este sistema al campo educativo de la electrónica, de ese modo, se contribuye en sistematizar información y resultados en pertinencia a estrategias de verificación del montaje de un 
sistema electrónico, sirviendo como referencia para el desarrollo de cursos académicos y de formación en universidades y centros especializados.

\section{Sistema Boundary-Scan (JTAG)}

El sistema de acuerdo a lo planteado por Ikor (2015), permite:

1. Realizar el testeo de varios chips Boundary-Scan, éstos normalmente se ponen en cascada $o$ en cadena de tal forma que el primer chip que comunica con el adaptador Boundary-Scan para PC también está conectado al segundo chip, este segundo chip está conectado con un tercer chip y así hasta el último chip que estará conectado éste último también al adaptador de PC para sacar los datos de todos los chips.

2. Otra de las ventajas del Boundary-Scan es que permite comprobar otros dispositivos o chips que no son Boundary-Scan como por ejemplo una memoria RAM. Si nos centramos en este caso, la memoria conectada a un microprocesador que sí es Boundary-Scan, permite que al cambiar y leer los puertos de E/S del microprocesador se está accediendo a dicha memoria RAM permitiendo escribir y leer datos en ella comprobando así su funcionamiento.

3. La comunicación con el dispositivo Boundary-Scan normalmente se hace con un adaptador que se conecta a un PC y al circuito electrónico en el que se ha provisto de un conector especial para el test Boundary-Scan. En el PC se ejecuta un programa o software de testeo en el cual se detecta el dispositivo o dispositivos Boundary-Scan conectados, se puede leer y cambiar el estado de las entradas y salidas, ejecutar secuencias de test, prueba e incluso programación de otros dispositivos no Boundary-Scan, etc.

Desde lo planteado, Sanabria, Pedraza, Aponte \& Vitola (2011), comentan que este sistema permite generar prototipos a bajo costo, lo cual es prudente en el sector universitario, por cuanto los programas a la formación en electrónica podrían contemplar 
la generación de experimentos en función del Boundary-Scan (JTAG), por consiguiente plantean que:

Como ingenieros, es importante el correcto diseño de los sistemas, pero de igual manera es indispensable ofrecer plataformas que faciliten la realización de pruebas de funcionamiento y que verifiquen el estado del circuito impreso así como de las soldaduras, daños en los componentes y demás circunstancias que puedan afectar el objetivo trazado (p. 46).

Siendo posible formar a los futuros ingenieros en competencias integrales que le permitan abordar en razón de las circunstancias académicas y tecnológicas, teniendo en cuenta además las innovaciones existentes en el sector tecnológico. Por otro lado, Moreno (2017) señala las bondades del

Hemos evaluado la mayoría de los sistemas líderes en el mercado, y hemos encontrado en XJTAG el ganador por una serie de razones, ofrece todas las características que necesitamos, igualando o excediendo los demás, pero a un precio mejor. También hemos encontrado apoyo técnico. XJTAG es el más accesible y sensible.

Las recomendaciones planteadas, indican la importancia que tiene el sistema para la empresa militar, siendo importante visualizar los aportes generados en favor de maximizar las operaciones de una industria que no trabaja con margen de error. Así mismo es empleado en diversos sistemas como los planteados por INTI (2017).

a. Este plug-in puede usarse con el siguiente software: OpenOCD (Linux), UrJTAG (Linux), Xilinx iMPACT (Linux). Probablemente pueda usarse con otros programas y/o sistemas operativos, las combinaciones mencionadas fueron verificadas y en los links encontrará detalles de configuración para cada software. Debido a que el FT2232H, implementa JTAG en forma nativa, y a que los programas mencionados pueden ser configurados (o al menos engañados...) este plug-in no necesita de ningún circuito complejo. La configuración mostrada en este esquemático fue usada exitosamente en todos los casos. Al no haber un consenso en cuanto al 


\section{CIENCIAMATRIA}

Revista Interdisciplinaria de Humanidades, Educación, Ciencia y Tecnología

Año V. Vol. V. N09. Julio - Diciembre 2019

Hecho el depósito de ley: pp201602FA4721

ISSN-L: 2542-3029; ISSN: 2610-802X

Universidad Nacional Experimental Francisco de Miranda (UNEFM). Santa Ana de Coro. Venezuela

Alba de los Cielos Miranda Villacis; Marlon Ernesto Moscoso Martínez; Miguel Andrés Sánchez Almeida

conector JTAG se decidió implementar este plug-in utilizando cables separados (flying leads):

JTAG Base. Tomado de INTI (2017).

Figura 1.

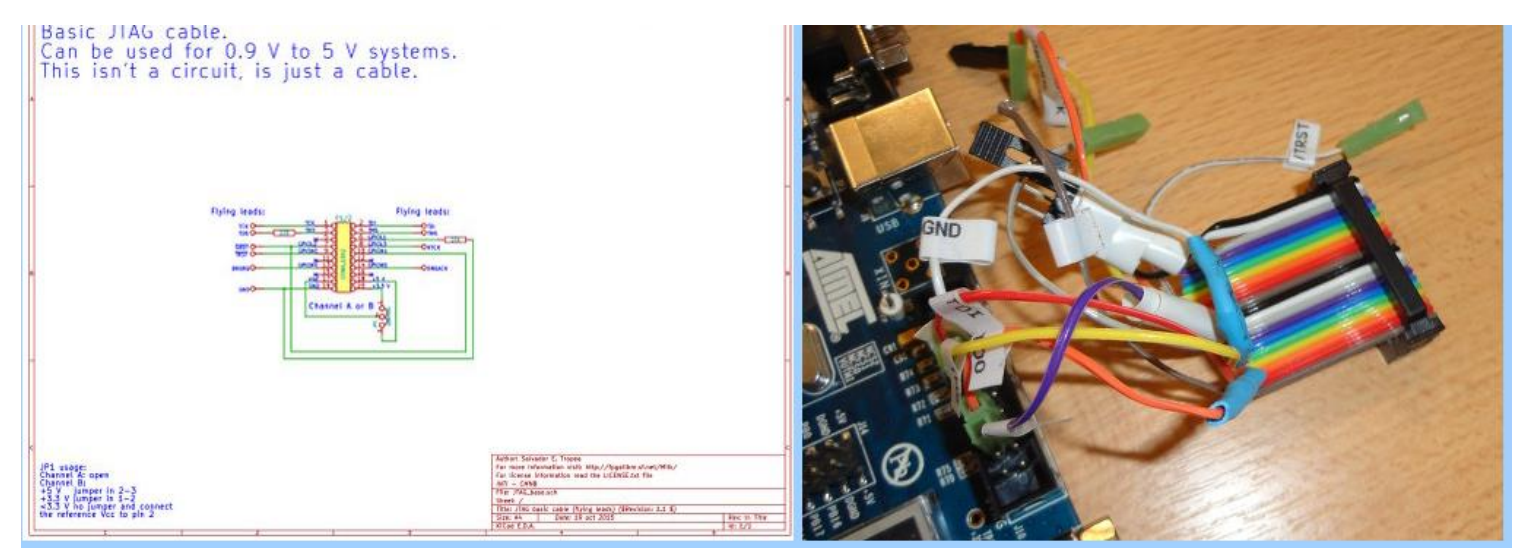

Otro escenario de entrenamiento JTAG, lo promueve ABI Electronics (2019), al indicar un prototipo que permite el entrenamiento de los usuarios, en este sentido:

1. La placa entrenadora JTAGMaster Training Board es una PCB diseñada para demostrar los principios de prueba y programación JTAG pero también para mostrar las capacidades de la unidad JTAGMaster junto a su software, AIMs. La placa ofrece lo siguiente:

a) Cuatro dispositivos digitales en una cadena JTAG

b) Cuatro pulsadores de botón para el control de la operación de la placa

c) Una pantalla LCD de 2 líneas de 16 caracteres cada una para el menú de selección

d) Cuatro canales analógicos de entrada

e) Dos canales analógicos de salida

f) Ocho canales de Entrada/Salida digitales 
2. El principal objetivo de la placa entrenadora JTAGMaster Training Board es enseñar a los usuarios como programar dispositivos en una cadena JTAG y como llevar a cabo pruebas "boundary scan" para comprobar una placa con éxito.

Entre los protocolos que promueve ABI Electronics (2019), se presentan:

- ATMEGA16 MICRO: EI ATMEGA16 es un ejemplo de un microcontrolador que puede ser programado y depurado (debugged) usando su interfaz JTAG. También soporta el "boundary scan" para que permitir a sus pines sean monotorizados por el JTAGMaster.

- ALTERA EP1CF324 FPGA: El Atera EP1C4F324 es un FPGA de propósito general que necesita ser reprogramado cada vez que la placa es alimentada. Esto se puede hacer a través de la configuración de memoria o del JTAGMaster.

- Memoria XCF10S: El Xilinx XCF10S Platform FLASH contiene 1Mbits de memoria y puede ser utilizado para programar dispositivos FPGA.

- XC95288XL CPLD: El Xilinx XC95288XL CPLD contiene su propia configuración de memoria y no necesita ser reprogramado cada vez que se activa la alimentación. Sin embargo, tiene menor capacidad que el Atera FPGA.

Prototipos similares podrían ser construidos en las universidades con la finalidad de formar a los futuros ingenieros en el diseño, construcción y manejo adecuado de sistemas que tengan como base JTAG.

\section{Estrategias para el aprendizaje JTAG}

La estrategia para el aprendizaje JTAG, es necesario basarla en la generación de proyectos de investigación, para esto se requiere que el estudiante sea formado en una visión integral desde una concepción metodológica, lo cual le permitirá generar cálculos cuantitativos, procesos experimentales, así como lo relacionado a lo cualitativo, en cuanto debe comprender que la tecnología es usada por personas con diversos propósitos, conocer estos propósitos proporciona la posibilidad de estructurar un acercamiento que 
permita indagar las necesidades y cualidades del usuario, de ese modo se podrá diseñar un sistema en relación a perspectivas individuales.

Aunado a lo descrito, la fusión de aprendizaje desde la investigación con enfoque mixto, permitirá al participante comprender el ecosistema de vida del ser humano, articulándose un pensamiento sistémico y ecológico, ante lo cual, Aldana Zavala \& Colina Ysea (2019), señalan que la educación actual es responsable de promover un estilo sustentable, aunado lo descrito, Aldana, Salón \& Guzmán (2019), destacan la necesidad de formar con pensamiento global para interconectar las múltiples realidades en una respuesta por parte del estudiante. Así mismo, lanni Gómez (2017), destaca la importancia de la investigación como centro neurálgico para el aprendizaje, siendo una tendencia en las universidades por cuanto esto es una demanda de las empresas, las cuales han aceptado la visión que el principal capital, se encuentra en el conocimiento. Rodríguez Acasio \& Colina Ysea (2016).

De ese modo, por medio de la realización de proyectos de investigación, el Aprendizaje Basado en Proyectos es un modelo de aprendizaje en el que los estudiantes planean, implementan y evalúan proyectos que tienen aplicación en el mundo real más allá del aula de clase". (Blank, 1997; Dickinson, et al, 1998; Harwell, 1997). La universidad politécnica de Madrid (2008) señala que:

Para el uso de esta técnica de aprendizaje es importante la coordinación de docentes de diferentes materias para que en un mismo proyecto los alumnos tengan que aplicar conocimientos de distintas asignaturas y que, además, los nuevos conocimientos que construyan formen parte de una estructura coherente e integrada (p. 2).

Así un estudiante durante un período académico puede construir un proyecto de aprendizaje en cooperación con compañeros y docentes de otras áreas o asignaturas, siendo necesario que la universidad flexibilice el currículo para que puedan desarrollar el proyecto en base a una determinada problemática o área a desarrollar, esto posibilita diagnosticar que necesidades requieren los clientes sobre JTAG. La universidad politécnica de Madrid (2008) señala que: 
Con este método los alumnos tienen que trabajar en equipo desde el principio y desarrollar un proyecto que solucione la situación presentada planificando la actuación, distribuyendo tareas, investigando, analizando los contextos involucrados, desarrollando el plan establecido, evaluando las posibles consecuencias, previendo los éxitos, etc. Exige, el AOP (aprendizaje orientado por proyectos), en todo momento que el alumno esté activo, interactuando con sus compañeros, contrastando opiniones, ideas, teorías, aplicaciones para llegar a consensos fundamentados y justificados, etc. La reflexión sobre el conocimiento con el fin de generar nuevo conocimiento es un eje central de esta metodología (p. 2).

El fomento de esta estrategia de aprendizaje, además permite que el estudiante se convierta en un investigador autónomo, constructor de su propio aprendizaje en cooperación de sus compañeros y asesores docentes. Así mismo, Aldana Zavala \& Colina Ysea (2017), destacan que los investigadores al trabajar bajo el enfoque de aprendizaje por proyectos, genera las siguientes competencias "Formación a lo largo de la vida. 2. Acompañamiento Pedagógico. 3. Investigará en conjunto con los participantes". Lo cual se traduce en:

1. Formación a lo largo de la vida: Desarrollada en los ambientes de aprendizaje y en las múltiples realidades socio-turística, mediante encuentros de socialización de los actores involucrados del proceso, con la finalidad de fijar la ruta a seguir durante el trayecto investigativo - académico,

2. Acompañamiento Pedagógico: Se generará el intercambio de saberes y conocimientos pertinentes para consolidar la estructura cognitiva de los participantes en temas que sean coherentes - necesarios de ser abordados para tener la base epistémica consolidada en el trabajo de campo,

3. Investigará en conjunto con los participantes: Es el trabajo de campo en donde se trabajará progresivamente en la resolución de un determinado "problema o foco", en una institución u organización turística, lo cual permite abordar en la vida real, los conocimientos generados en el punto 2. (p. 22). 
Así el diseño de sistemas electrónicos pueden ser trabajados desde una filosofía de gestión educativa basada en la investigación, redundando en formar investigadores para la vida, es decir que no solo la realicen para aprobar su formación académica en la universidad, sino, que puedan aportar continuamente a la solución de los problemas sociales en relación a lo tecnológico, más aun, cuando las ciencias de la robótica se manejan desde una perspectiva multidisciplinar.

\section{REFLEXIONES DE CIERRE}

El sistema JTAG se torna versátil por su aplicabilidad en el mundo de la tecnología y dispositivos digitales, permitiendo realizar análisis de recuperación de información encriptada, siendo esta una necesidad en la sociedad global, así como en disciplinas como la forense donde se requiere recuperar datos de dispositivo móviles y otros hardware de almacenamiento con la finalidad de procesar datos para el procesamiento legal, esto abre la posibilidad que las universidades formen a sus estudiantes en diseñar, construir, sistemas JTAG, lo cual es viable de acuerdo a las experiencias presentadas en la revisión del actual manuscrito.

Se requiere de incorporar nuevas estrategias de aprendizaje que permitan construir prototipos en conformidad a los requerimientos del mercado y del presupuesto de la institución, para lo cual se puede implementar el aprendizaje basado por proyectos por cuanto este permite aprender haciendo e investigando desde un enfoque cooperativo, lo que además permite consolidar a un investigador para la vida, es decir, que investigará para ser un profesional en capacidad de brindar respuestas desde el contexto donde se encuentre y no solo para cumplir con un proceso de formación académica.

El mundo de los sistemas JTAG, abre la posibilidad de transcender la visión educativa tradicional para trabajar por proyectos, esto implica que se pueden conformar equipos de trabajo en la universidad, donde puedan participar diversos docentes y estudiantes, bridando una noción multidisciplinaria al proyecto, de ese modo, se promueve el aprendizaje significativo desde la construcción de prototipos, los cuales además podrían 
ser abordados en función de trabajar el enfoque global sustentable, situación que además permite formar en valores, consolidando un ciudadano reflexivo, de ese modo, la educación y tecnología se conjugan para contribuir al buen vivir de la sociedad ecuatoriana.

\section{REFERENCIAS CONSULTADAS}

1. Aldana Zavala, J., \& Colina Ysea, F. (2019). Marketing verde en la conformación de una ciudadanía planetaria en el ámbito educativo latinoamericano. Revista San Gregorio, 0(31), 150-161. doi:http://dx.doi.org/10.36097/rsan.v0i31.972

2. Aldana, J., Salón, M., \& Guzmán, N. (2019). Liderazgo sistémico en las competencias gerenciales docentes universitarias. CIENCIAMATRIA, 5(8), 50-74. Recuperado a partir http://cienciamatriarevista.org.ve/index.php/cm/article/view/87

3. Aldana Zavala, J.J. \& Colina Ysea, F. (2017). Reingeniería de los programas de turismo de postgrado UNEFM. Revista Ciencias Sociales y Educativa UNEFM, 7(4), 12-23. Recuperado de https://rcseunefm.files.wordpress.com/2018/03/rcsevolviinro4-final1.pdf

4. Ianni Gómez., L. (2017). MIRAMDA: Una propuesta educativa emergente desde la investigación. Revista Arbitrada Interdisciplinaria Koinonía, 2(3), 9-30. Recuperado de http://fundacionkoinonia.com.ve/ojs/index.php/revistakoinonia/article/view/49/36

5. Blank, W. (1997). Authentic instruction. In W.E. Blank \& S. Harwell (Eds.), Promising practices for connecting high school to the real world (pp. 15-21). Tampa, FL: University of South Florida. (ERIC Document Reproduction Service No. ED407586)

6. Ikor (2015). Boundary-Scan (JTAG). Recuperado de https://blog.ikor.es/boundaryscan/

7. INTI (2017). JTAG. http://fpgalibre.sourceforge.net/Milk/milk jtag.html

Recuperado de

8. Moreno, D. (2017). TECNOBIT especialista español en Defensa y Espacio, optimiza las ventajas del "Boundary Scan" con XJTAG. Recuperado de https://www.waltergallegos.com/pdf/xjtag tecnobit estudio-de-caso.pdf 
9. Rodríguez Acasio, F., \& Colina Ysea, F. (2016). La investigación desde la gestión del conocimiento en el contexto de las Universidades Nacionales Experimentales. Revista Arbitrada Interdisciplinaria Koinonía, 1(1), 88-100. Recuperado de http://fundacionkoinonia.com.ve/ojs/index.php/revistakoinonia/article/view/17/10

10. Sanabria, C. Pedraza, A. Aponte, J. Vitola (2011). JTAG: conceptos y diseño de una herramienta para FPGAs. Recuperado de revistas.usantotomas.edu.co , index.php > intekhnia > article > download

11. Universidad politécnica de Madrid (2008). Aprendizaje orientado por proyectos. http://innovacioneducativa.upm.es/guias/AP PROYECTOS.pdf

\section{REFERENCES CONSULTED}

1. Aldana Zavala, J., \& Colina Ysea, F. (2019). Green marketing in the conformation of a planetary citizenship in the Latin American educational field. San Gregorio Magazine, 0 (31), 150-161. doi: http: //dx.doi.org/10.36097/rsan.v0i31.972

2. Aldana, J., Salón, M., \& Guzmán, N. (2019). Systemic leadership in university teaching management skills. SCIENCE, 5 (8), 50-74. Recovered from http://cienciamatriarevista.org.ve/index.php/cm/article/view/87

3. Aldana Zavala, J.J. \& Colina Ysea, F. (2017). Reengineering of UNEFM postgraduate tourism programs. UNEFM Social and Educational Sciences $\begin{array}{lllll}\text { Magazine, } & 7 & \text { (4), } & \text { 12-23. } & \text { Recovered }\end{array}$ https://rcseunefm.files.wordpress.com/2018/03/rcsevolviinro4-final1.pdf

4. Ianni Gómez., L. (2017). MIRAMDA: An educational proposal emerging from research. Interdisciplinary Arbitrated Review Koinonía, 2 (3), 9-30. Recovered from http://fundacionkoinonia.com.ve/ojs/index.php/revistakoinonia/article/view/49/36

5. Blank, W. (1997). Authentic instruction. In W.E. Blank \& S. Harwell (Eds.), Promising practices for connecting high school to the real world (pp. 15-21). Tampa, FL: University of South Florida. (ERIC Document Reproduction Service No. ED407586)

6. Ikor (2015). Boundary-Scan (JTAG). Recovered from https://blog.ikor.es/boundaryscan/ 


\section{CIENCIAMATRIA}

Revista Interdisciplinaria de Humanidades, Educación, Ciencia y Tecnología

Año V. Vol. V. N09. Julio - Diciembre 2019

Hecho el depósito de ley: pp201602FA4721

ISSN-L: 2542-3029; ISSN: 2610-802X

Universidad Nacional Experimental Francisco de Miranda (UNEFM). Santa Ana de Coro. Venezuela

Alba de los Cielos Miranda Villacis; Marlon Ernesto Moscoso Martínez; Miguel Andrés Sánchez Almeida

7. INTI

(2017).

JTAG

Recovered

from http://fpgalibre.sourceforge.net/Milk/milk jtag.html

8. Moreno, D. (2017). TECNOBIT Spanish specialist in Defense and Space, optimizes the advantages of "Boundary Scan" with XJTAG. Recovered from https://www.waltergallegos.com/pdf/xjtag tecnobit estudio-de-caso.pdf

9. Rodríguez Acasio, F., \& Colina Ysea, F. (2016). Research from knowledge management in the context of National Experimental Universities. Interdisciplinary Arbitrated Review Koinonía, 1 (1), 88-100. Recovered from http://fundacionkoinonia.com.ve/ojs/index.php/revistakoinonia/article/view/17/10

10. Sanabria, C. Pedraza, A. Aponte, J. Vitola (2011). JTAG: concepts and design of a tool for FPGAs. Recovered from journals.usantotomas.edu.co sindex.phps intekhnia sarticles download

11.Polytechnic University of Madrid (2008). Project oriented learning. http://innovacioneducativa.upm.es/guias/AP PROYECTOS.pdf 